\title{
Improvement of solar-cycle prediction: Plateau of solar axial dipole moment
}

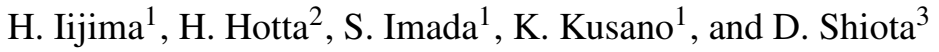 \\ ${ }^{1}$ Division for Integrated Studies, Institute for Space-Earth Environmental Research, Nagoya University, Furocho, Chikusa-ku, \\ Nagoya, Aichi 464-8601, Japan \\ e-mail: h.iijima@isee.nagoya-u.ac.jp \\ 2 Department of Physics, Faculty of Science, Chiba University, 1-33 Yayoi-chou, Inage-ku, Chiba 263-8522, Japan \\ 3 Applied Electromagnetic Research Institute, National Institute of Information and Communications Technology (NICT), \\ 4-2-1 Nukui-Kitamachi, Koganei, Tokyo 184-8795, Japan
}

Received 22 August 2017 / Accepted 11 October 2017

\section{ABSTRACT}

\begin{abstract}
Aims. We report the small temporal variation of the axial dipole moment near the solar minimum and its application to the solar-cycle prediction by the surface flux transport (SFT) model.

Methods. We measure the axial dipole moment using the photospheric synoptic magnetogram observed by the Wilcox Solar Observatory (WSO), the ESA/NASA Solar and Heliospheric Observatory Michelson Doppler Imager (MDI), and the NASA Solar Dynamics Observatory Helioseismic and Magnetic Imager (HMI). We also use the SFT model for the interpretation and prediction of the observed axial dipole moment.

Results. We find that the observed axial dipole moment becomes approximately constant during the period of several years before each cycle minimum, which we call the axial dipole moment plateau. The cross-equatorial magnetic flux transport is found to be small during the period, although a significant number of sunspots are still emerging. The results indicate that the newly emerged magnetic flux does not contribute to the build up of the axial dipole moment near the end of each cycle. This is confirmed by showing that the time variation of the observed axial dipole moment agrees well with that predicted by the SFT model without introducing new emergence of magnetic flux. These results allow us to predict the axial dipole moment at the Cycle 24/25 minimum using the SFT model without introducing new flux emergence. The predicted axial dipole moment at the Cycle $24 / 25$ minimum is $60-80$ percent of Cycle 23/24 minimum, which suggests the amplitude of Cycle 25 is even weaker than the current Cycle 24.

Conclusions. The plateau of the solar axial dipole moment is an important feature for the longer-term prediction of the solar cycle based on the SFT model.
\end{abstract}

Key words. Sun: activity - Sun: photosphere - sunspots

\section{Introduction}

The prediction of the 11-year sunspot cycle is an important task for the long-term prediction of the space weather. Various methods have been suggested on this subject (Hathaway et al. 1999; Petrovay 2010; Pesnell 2012, 2016). However, the predictability of these methods is still controversial, especially in terms of the prediction of weak sunspot activity in Cycle 24 . One of the most successful approaches is the precursor method (e.g., Ohl 1966; Schatten et al. 1978; Svalgaard et al. 2005; Schatten 2005; Wang \& Sheeley 2009; Muñoz-Jaramillo et al. 2013). This method uses the strength of the polar magnetic field on the solar surface (or relating indices like the axial dipole moment and geomagnetic activity index) at the solar cycle minimum as an indicator. This indicator has a high correlation with the amplitude of the next cycle maximum. The precursor method allows us to predict the next solar cycle amplitude approximately five years before the cycle maximum.

Recently, the prediction of the polar field at the Cycle 24/25 minimum was carried out based on the surface flux transport (SFT) models (Upton \& Hathaway 2014b; Cameron et al. 2016; Hathaway \& Upton 2016); they assume empirical modelings to predict the newly emerging magnetic flux until the solar cycle minimum. As noted by Jiang et al. (2014, 2015), modeling of the new flux emergence causes considerable uncertainty (or dispersion) of the resulting axial dipole moment. The accuracy and validity of the emerging flux modeling is crucial for the prediction of the future solar cycle. In this study, we investigate the time evolution of the observed axial dipole moment to explore the better modeling of the new flux emergence in the SFT model. We also carry out the prediction of the next solar cycle amplitude based on the observation and the simulation by the SFT model.

\section{Observation}

We use the line-of-sight synoptic magnetogram taken from the ESA/NASA Solar and Heliospheric Observatory Michelson Doppler Imager (MDI, Scherrer et al. 1995), the NASA Solar Dynamics Observatory Helioseismic and Magnetic Imager (HMI, Scherrer et al. 2012), and the Wilcox Solar Observatory (WSO). The line-of-sight magnetic field is projected to the radial component of the magnetic field assuming that the photospheric magnetic field is vertical. The magnetic field strength of the WSO data is multiplied by a factor of two so as to account for the saturation of the magnetic signal. We also use the monthly sunspot number provided by WDC-SILSO.

The thick solid lines in Fig. 1 show the time evolution of the observed axial dipole moment in Cycles 21, 22, 23, and 24. 

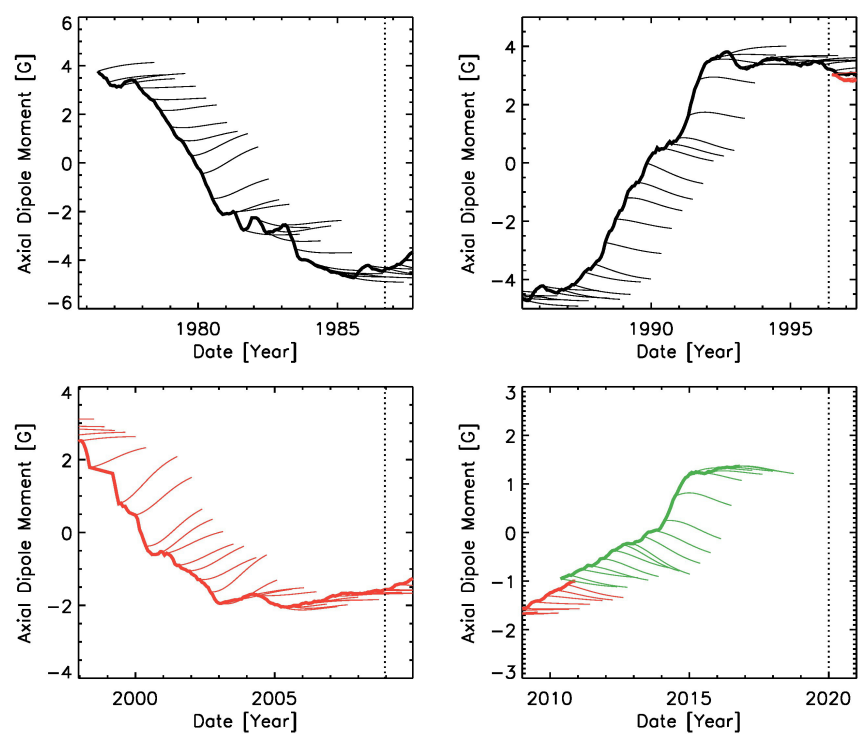

Fig. 1. Time evolution of the observed and predicted axial dipole moment. The thick lines indicate the observed axial dipole moment (black: WSO, red: MDI, green: HMI). The thin lines indicate the prediction by the SFT without introducing new emerging flux starting from the observed synoptic magnetogram. The dotted lines indicate the timing of the cycle minimum. The minimum in Cycle 24 is assumed to be in 2020 .

As a precursor of the next solar cycle amplitude, we use the axial dipole moment $D=3 /(4 \pi) \int_{4 \pi} B_{R} \sin \lambda \mathrm{d} \Omega$, where $B_{R}$ is the radial component of the magnetic field and $\lambda$ is the latitude. We find that the last three cycles (21-23) have periods (or plateaus) in which the time evolution of the axial dipole moment becomes very small near the end of each cycle. This plateau continues for at least approximately three years before each sunspot cycle minimum. It is also notable that the plateau in Cycle 23 continues for an exceptionally long time (from 2004 to 2009), which is known as the extended cycle minimum (e.g., Upton \& Hathaway 2014a; Jiang et al. 2015). We explain the thin solid lines in Sect. 3.

The cross-equatorial transport of the net magnetic flux plays a crucial role for the build-up of the axial dipole moment at the solar minimum (Cameron et al. 2013). The temporal change of the axial dipole moment is affected by the poleward transport of the active region and the cross-equatorial transport of the magnetic flux. On the other hand, the permanent change of the axial dipole moment at the cycle minimum, in which a very small amount of the magnetic flux remains in low latitude, is only produced by the cross-equatorial flux transport.

We define the cross-equatorial flux transport as the time derivative of the net magnetic flux. The net magnetic flux in the northern hemisphere is defined as

$\Phi^{N}=R_{\odot}^{2} \int_{0}^{\pi / 2} \cos \lambda \mathrm{d} \lambda \int_{-\pi}^{\pi} \mathrm{d} \phi B_{R}(\lambda, \phi)$,

where $\phi$ is the longitude. Because the magnetic monopole is not allowed, the net flux in the northern hemisphere $\Phi^{N}$ exactly balances with the net flux in the southern hemisphere. Thus, no hemispheric asymmetry exists in the net hemispheric flux. The cross-equatorial transport of the net magnetic flux per unit time is computed by the time derivative of the net flux $\mathrm{d} \Phi^{N} / \mathrm{d} t$.

Figure 2 shows the comparison between the cross-equatorial flux transport (solid lines) and the monthly total sunspot number (dashed lines). We find a significant number of sunspots (several
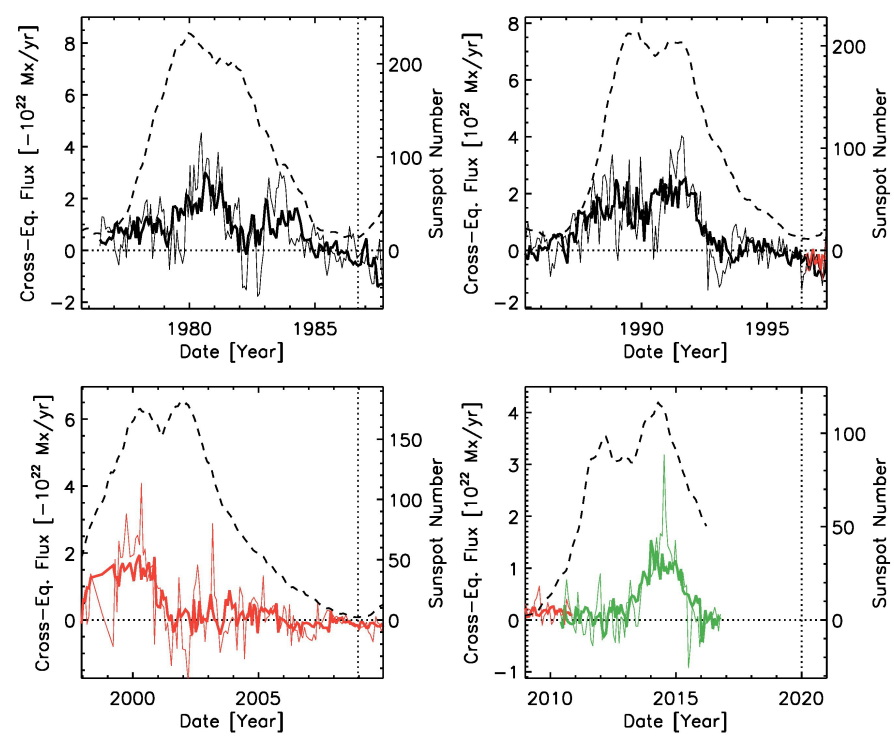

Fig. 2. Time variation of the 13-month smoothed monthly total sunspot number (dashed lines) and the cross-equatorial flux transport (solid lines) in each sunspot cycle. The definition of the cross-equatorial flux transport is described in the body text. The cross-equatorial flux transport is smoothed by taking the moving average of 1 year (thin) and 2 years (thick). The dotted lines indicate the timing of the cycle minimum. The minimum in Cycle 24 is assumed to be in 2020 .

tens of percents of the maximum) emerging during the plateau of the axial dipole moment (approximately 3 years before each minimum; Fig. 1). However, the cross-equatorial flux transport is nearly zero during the axial dipole moment plateau (typically 3 years before each minimum; Fig. 1). This suggests that the newly emerged sunspots do not contribute to the variation of the axial dipole moment during the period. This is surprising because the sunspots appear at low latitude in the latter phase of the sunspot cycle, which makes the cross-equatorial flux transport easily distinguishable. We note that the cross-equatorial flux based on the Kitt Peak Vacuum Telescope of the US National Solar Observatory (NSO) has larger time variation before the mid-1990s (see Fig. 5 in Cameron et al. 2013) than that in the WSO data. This difference makes it difficult to identify the existence of the axial dipole moment plateau near the end of Cycle 21 in the NSO data. Further analysis should be undertaken on the difference of the observational instruments. In the following Section, we further show that the time evolution of the observed axial dipole moment near the end of each cycle is well modeled by the SFT model without introducing the new flux emergence.

\section{Modeling and prediction}

Following the observational results in Sect. 2, we try to model the time evolution of the axial dipole moment by the SFT model without introducing the new flux emergence. We model the time evolution of the photospheric radial magnetic field based on the SFT model (e.g., Sheeley et al. 1983; DeVore et al. 1984; Wang et al. 1989; van Ballegooijen et al. 1998). The basic equation of the SFT code, that is, the two-dimensional advectiondiffusion equation, is azimuthally averaged to get the onedimesional SFT equation (e.g., Cameron \& Schüssler 2007). The equation is written as

$$
\frac{\partial B_{R}}{\partial t}+\frac{1}{R_{\odot} \sin \theta} \frac{\partial}{\partial \theta}\left(B_{R} V_{\theta} \sin \theta\right)=\frac{1}{R_{\odot}^{2} \sin \theta} \frac{\partial}{\partial \theta}\left(\eta \sin \theta \frac{\partial B_{R}}{\partial \theta}\right),
$$


where $\theta$ is the colatitude. The meridional flow $V_{\theta}$ is taken from van Ballegooijen et al. (1998). The turbulent magnetic diffusivity $\eta$ is assumed to be $250 \mathrm{~km}^{2} \mathrm{~s}^{-1}$ (e.g., Cameron et al. 2016). This one-dimensional SFT equation can describe the evolution of the azimuthally averaged magnetic field that is analytically identical to the original two-dimensional SFT equation with the longitudinally and temporally constant turbulent diffusivity, meridional flow, and differential rotation. Because we only focus on the azimuthally averaged axial dipole moment or the axial magnetic dipole moment, this one-dimensional SFT equation is sufficient for this study. The equation is solved by the secondorder central finite difference scheme in space and the secondorder Strong Stability Preserving Runge-Kutta method in time. The advection term is stabilized only by the turbulent magnetic diffusion and no additional artificial diffusion is used. The latitudinal grid size is $5.5 \mathrm{Mm}$ in this study.

The thin solid lines in Fig. 1 show the simulations of our SFT model (Eq. (2)) that do not include the contribution of the new flux emergence. Each SFT simulation is started from each snapshot of the longitudinally averaged synoptic magnetogram and integrated for 5 years. Near the cycle maximum, the observed axial dipole moment greatly changes in time and changes the sign of the axial dipole moment. On the other hand, the axial dipole moment predicted by the SFT evolves toward to the value in the preceding minimum. This behavior is caused because the independent tilted active region contributes to the global axial dipole moment (e.g., Wang \& Sheeley 1991; Jiang et al. 2014; Yeates et al. 2015). When the latitude of the emergence is high enough, this contribution is transient and eliminated by the poleward transport of the active region. When the active region emerged at low latitude (as in the latter part of the solar cycle), the magnetic flux of the preceding polarity can be transported across the equator. This contribution is not canceled by the poleward transport. This feature is shown more clearly in the HMI/MDI data than in the WSO data in which small active regions are flattened (or averaged) by the lower spatial resolution. During the plateau of the axial dipole moment near the end of each cycle, the time variation of the predicted dipole moment also becomes small. The simulated axial dipole moment shows good agreement with the observed dipole moment. The small time variation of the axial dipole moment during the plateau indicates small cross-equatorial transport of the magnetic flux. Although we do not show the WSO data in Cycle 23 and 24 for the visibility of Fig. 1, we note that the observed and predicted axial dipole moments of the WSO data also exhibit characteristics similar to the MDI/HMI data.

Under the assumption that the contribution of the emerging flux is negligibly small in the period of several years before each cycle minimum, we predict the future axial dipole moment and the cycle amplitude with the SFT model without the new flux emergence. As shown in Fig. 2, the cross-equatorial flux transport in Cycle 24 becomes approximately zero from the beginning of 2016. This result allows us to predict the axial dipole moment at the Cycle 24/25 minimum from the current observation. Figure 3 shows the amplitude of the solar sunspot cycle as a function of the three-year prediction of the axial dipole moment at the cycle minimum. The amplitude of each cycle is measured by the 13-month smoothed monthly total sunspot number. The total sunspot number is averaged over the six months before and after the cycle maximum. The predicted axial dipole moment at the cycle minimum is simulated from the synoptic magnetogram 3 years before the solar minimum by the SFT neglecting new emergence of magnetic flux. The axial dipole moment is averaged over the six months before and after the

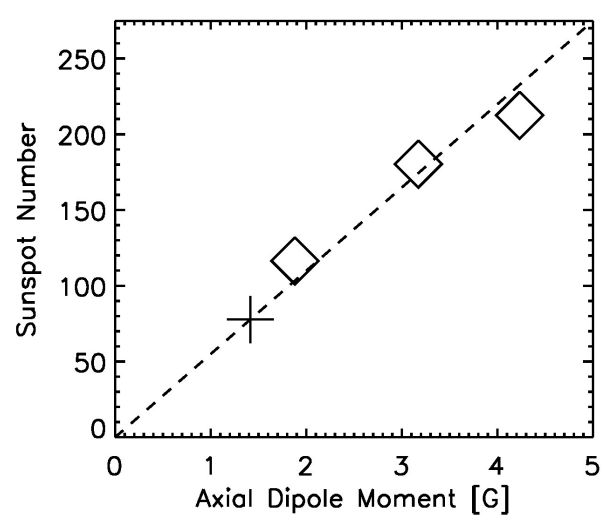

Fig. 3. Maximum value of the 13-month smoothed monthly total sunspot number in each sunspot cycle (Cycle 22, 23, and 24; diamond) and the predicted cycle amplitude in Cycle 25 (cross) as a function of the axial dipole moment at the previous minimum predicted from the magnetogram observed three years before the minimum. The least-square fit for Cycles 22, 23, and 24 that crosses the point of origin is shown as the dashed line. The correlation coefficient for Cycles 22, 23, and 24 is 0.99 .

timing of 3 years before the solar minimum. At the Cycle 23/24 minimum (at the end of Cycle 23) and Cycle 24/25 minimum, multiple data sources (WSO, MDI, and HMI) are available. In such cases, we use the average value of the axial dipole moments independently predicted from the available data sources. The cycle maximum/minimum is defined as the date on which the 13-month smoothed monthly total sunspot number becomes maximum/minimum.

The predicted axial dipole moment is highly correlated to the amplitude of the next cycle with a correlation coefficient of 0.99 for Cycles 22, 23, and 24. This proves the predictability of the future solar cycle using our method. We assume the proportional relation between the predicted axial dipole moment of the minimum and the maximum sunspot number in the next cycle and apply the least-square fit for Cycles 22, 23, and 24. Based on the relation, we predict that the maximum total sunspot number in Cycle 25 will be 60-80 percent of Cycle 24 . The error range of the predicted value comes from the averaging procedure to measure the total sunspot number and the axial dipole moment, the deviation from the proportional relation between them, and the difference among the multiple instruments. We also note that the deviation of the observed axial dipole moment exists between the WSO $(\sim 1.77 \mathrm{G})$ and HMI $(\sim 1.39 \mathrm{G}$; bottom right panel in Fig. 1) data in 2015-2017 as a source of the prediction error.

\section{Discussion}

In this study, we show that the solar cycles 21-24 have the common characteristic that the axial dipole moment hardly changes during the period near the end of each cycle, which we call the axial dipole moment plateau. The cross-equatorial flux transport becomes very small during the period. This is also confirmed by showing that the time evolution of the axial dipole moment is well described by the simplified SFT model without the new flux emergence. The axial dipole moment predicted by the SFT model shows high correlation to the amplitude in the next cycle, which allows us to predict the amplitude of Cycle 25.

We get a high correlation between the axial dipole moment predicted three years before the cycle minimum and the amplitude of the next cycle maximum. We note that the similar high 
correlation is achieved between the axial dipole moment three years before the cycle minimum and the amplitude of the next solar cycle. This high correlation comes from the existence of the plateau near the end of each solar cycle. The result indicates the importance of the plateau for the prediction of the future axial dipole moment and the solar activity. Muñoz-Jaramillo et al. (2013) reported that the cycle prediction based on the polar field measurement performs well up to two years before minimum, after which the success rate drops dramatically. We further emphasize this point and show that, although a significant number of sunspots are still observed, the new emergence of the magnetic flux does not contribute to the polar field near the end of the cycle.

We predict that the strength of the axial dipole moment at the Cycle 24/25 minimum will be several tens of percent weaker than the previous minimum. This value is comparable but weakest among the predictions based on the other SFT models (Upton \& Hathaway 2014b; Cameron et al. 2016; Hathaway \& Upton 2016). It is natural that we get the weakest predicted value because we neglect the contribution of the new emergence of the magnetic flux in the simulation.

We find a plateau of the axial dipole moment before the cycle minimum for the last three cycles. The duration of the plateau ranges from $\sim 3$ years in Cycle 21 to $\sim 6$ years in Cycle 23. Although all of the cycles investigated in this study have the plateau lasting several years, we cannot deny the possibility that the last three cycles were special cases in the long history of the sunspot cycles. Muñoz-Jaramillo et al. (2012) reported the polar magnetic field estimated from the polar faculae measurements over 100 years. Their estimate of the polar field suggests that not all of the cycles in this time period show the plateau reported in our study. Because the difference between the temporal evolution of the faculae count and the axial dipole moment is significant, we need further studies to clarify the universality of the plateau.

The physical origin of the axial dipole moment plateau is not clear at present. Because a significant amount of the sunspots appear during the plateau, we need explanations for such small cross-equatorial flux transport during the period. The anomalously weak polar field and the extended minimum at the end of Cycle 23 has been studied by various authors (see also a review by Petrie 2015). One explanation of the small polar field at Cycle 23/24 minimum is that the emergence of the active region with the small tilt angle or the opposite polarity causes the source of the small cross-equatorial flux transport (Jiang et al. 2015). The other explanation is the variation of the meridional flow. The converging motion of the meridional flow in the activity band or the active region inflow (e.g., Haber et al. 2002; Zhao \& Kosovichev 2004; Hathaway \& Rightmire 2010) in the low latitude will prevent the cross-equatorial transport of the magnetic flux (e.g., De Rosa \& Schrijver 2006; Cameron \& Schüssler 2012; Martin-Belda \& Cameron 2017). The high gradient of the meridional flow near the equator is also a possible origin of the small cross-equatorial flux transport (Schrijver \& Liu 2008; Wang et al. 2009; Jiang et al. 2013). Although the meridional flow variation at the Cycle 23/24 minimum is not suitable to explain the observed axial dipole moment (Upton \& Hathaway 2014a), the effect on the flux transport should be considered to explain the plateau.

The deviation from the SFT model is also a possible origin of the axial dipole moment plateau. In the SFT model, the only effect of meridional flow is the horizontal (poleward) transport of the surface magnetic field. However, some studies indicated the possible role of the vertical (radial) component of the meridional circulation for the evolution of the surface magnetic field (e.g., Dikpati \& Choudhuri 1994; Hazra et al. 2017). On the other hand, Cameron et al. (2012) suggested that the effect of the vertical flow of the meridional circulation is suppressed when sufficiently strong downward magnetic pumping exists near the solar surface. Although the actual strength of the magnetic pumping near the solar surface is not well known, Stein \& Nordlund (2003) suggested that the convective motion near the surface will expel the magnetic flux rapidly under the strong density stratification, which implies strong magnetic pumping. Further studies are required for the quantitative evaluation of the effect of the multi-dimensional meridional flow on the evolution of the surface magnetic field.

Acknowledgements. We would like to thank the anonymous referee for insightful comments and suggestions. Wilcox Solar Observatory data used in this study were obtained via the web site http://wso.stanford.edu. The Wilcox Solar Observatory is currently supported by NASA. SOHO is a project of international cooperation between ESA and NASA. The SDO/HMI data used are courtesy of NASA and the SDO/HMI team. The sunspot records are courtesy of WDC-SILSO, Royal Observatory of Belgium, Brussels. This work is supported by MEXT/JSPS KAKENHI Grant Number 15H05816. H.H. is supported by MEXT/JSPS KAKENHI Grant Number JP16K17655, JP16H01169. This work was carried out by the joint research program of the Institute for Space-Earth Environment Research (ISEE), Nagoya University.

\section{References}

Cameron, R., \& Schüssler, M. 2007, ApJ, 659, 801

Cameron, R. H., \& Schüssler, M. 2012, A\&A, 548, A57

Cameron, R. H., Schmitt, D., Jiang, J., \& Işı1k, E. 2012, A\&A, 542, A127

Cameron, R. H., Dasi-Espuig, M., Jiang, J., et al. 2013, A\&A, 557, A141

Cameron, R. H., Jiang, J., \& Schüssler, M. 2016, ApJ, 823, L22

De Rosa, M. L., \& Schrijver, C. J. 2006, in ESA SP, 624, 12.1

DeVore, C. R., Boris, J. P., \& Sheeley, Jr., N. R. 1984, Sol. Phys., 92, 1

Dikpati, M., \& Choudhuri, A. R. 1994, A\&A, 291, 975

Haber, D. A., Hindman, B. W., Toomre, J., et al. 2002, ApJ, 570, 855

Hathaway, D. H., \& Rightmire, L. 2010, Science, 327, 1350

Hathaway, D. H., \& Upton, L. A. 2016, J. Geophy. Res., 121, 10

Hathaway, D. H., Wilson, R. M., \& Reichmann, E. J. 1999, J. Geophys. Res., 104, 22

Hazra, G., Choudhuri, A. R., \& Miesch, M. S. 2017, ApJ, 835, 39

Jiang, J., Cameron, R. H., Schmitt, D., \& Schüssler, M. 2013, Space Sci. Rev., 176,289

Jiang, J., Cameron, R. H., \& Schüssler, M. 2014, ApJ, 791, 5

Jiang, J., Cameron, R. H., \& Schüssler, M. 2015, ApJ, 808, L28

Martin-Belda, D., \& Cameron, R. H. 2017, A\&A, 597, A21

Muñoz-Jaramillo, A., Sheeley, N. R., Zhang, J., \& DeLuca, E. E. 2012, ApJ, 753,146

Muñoz-Jaramillo, A., Balmaceda, L. A., \& DeLuca, E. E. 2013, Phys. Rev. Lett., 111,041106

Ohl, A. 1966, Soln. Dannye, 12, 84

Pesnell, W. D. 2012, Sol. Phys., 281, 507

Pesnell, W. D. 2016, Space Weather, 14, 10

Petrie, G. J. D. 2015, Liv. Rev. Sol. Phys., 12, 5

Petrovay, K. 2010, Liv. Rev. Sol. Phys., 7, 6

Schatten, K. 2005, Geophys. Res. Lett., 32, L21106

Schatten, K. H., Scherrer, P. H., Svalgaard, L., \& Wilcox, J. M. 1978,

Geophys. Res. Lett., 5, 411

Scherrer, P. H., Bogart, R. S., Bush, R. I., et al. 1995, Sol. Phys., 162, 129

Scherrer, P. H., Schou, J., Bush, R. I., et al. 2012, Sol. Phys., 275, 207

Schrijver, C. J., \& Liu, Y. 2008, Sol. Phys., 252, 19

Sheeley, Jr., N. R., Boris, J. P., Young, Jr., T. R., DeVore, C. R., \& Harvey, K. L. 1983, in IAU Symp., 102, 273

Stein, R. F., \& Nordlund, A. 2003, in IAU Symp., 210, 169

Svalgaard, L., Cliver, E. W., \& Kamide, Y. 2005, Geophys. Res. Lett., 32, L01104

Upton, L., \& Hathaway, D. H. 2014a, ApJ, 792, 142

Upton, L., \& Hathaway, D. H. 2014b, ApJ, 780, 5

van Ballegooijen, A. A., Cartledge, N. P., \& Priest, E. R. 1998, ApJ, 501, 866

Wang, Y.-M., \& Sheeley, Jr., N. R. 1991, ApJ, 375, 761

Wang, Y.-M., \& Sheeley, N. R. 2009, ApJ, 694, L11

Wang, Y.-M., Nash, A. G., \& Sheeley, Jr., N. R. 1989, Science, 245, 712

Wang, Y.-M., Robbrecht, E., \& Sheeley, Jr., N. R. 2009, ApJ, 707, 1372

Yeates, A. R., Baker, D., \& van Driel-Gesztelyi, L. 2015, Sol. Phys., 290, 3189

Zhao, J., \& Kosovichev, A. G. 2004, ApJ, 603, 776 